\title{
Vector Superfields and Lorentz Violation
}

\author{
Don Colladay ${ }^{*}$ and Patrick McDonald \\ New College of Florida \\ 5800 Bay Shore, Rd, \\ Sarasota, FL 34243
}

(Dated: December 1, 2018)

\begin{abstract}
We extend Lorentz-violating Supersymmetry models to include vector superfields. The CPTpreserving model generalizes easily, while the obvious attempt at generalizing the CPT-violating model meets serious obstructions. Generalizations of the CPT-preserving but Lorentz-Violating model to higher dimensions are also straightforward. Compactification is used to reduce the six-dimensional theory to an $\mathcal{N}=2$ Lorentz-violating theory in four dimensions, while the tendimensional theory is used to produce an $\mathcal{N}=4$ Lorentz-violating theory. This may be useful in future constructions involving Ads/CFT correspondence with Lorentz violation.
\end{abstract}

PACS numbers: 11.30.Cp, 11.30.Pb,

\section{INTRODUCTION}

The conventional approach to applying supersymmetric theories to nature is to first extend the Poincaré symmetry to a superalgebra, then break the supersymmetry to reproduce something resembling the standard model at low energies. Alternatively, one may consider preserving the supersymmetry and breaking the Lorentz symmetry of the theory. Attempts have been made in this direction using two main approaches. The first involves perturbing the superalgebra sector involving $Q$ and $P^{\mu}$ operators, while the second involves keeping this sector of the superalgebra unperturbed. In the second case, it is argued that it is not possible to include any operators into the minimal supersymmetric extension of the Standard Model with dimension four or less [1]. There is also a third approach in which the SUSY is explicitly broken along with the Lorentz symmetry by the background fields present in the supermultilplets[2].

The first case in which the superalgebra is modified is explored in the current manuscript. Previous work along these lines began with the chiral superfield model by Berger and Kostelecký [3] in which the derivative operator was twisted in various ways to produce CPTpreserving and CPT-violating models of supersymmetry with Lorentz Violation. In the current manuscript, these models are extended to the vector superfield in higher dimensions, and nonabelian theories. The CPT-preserving model generalizes with minimal difficulty, while the CPTviolating version of the theory meets serious impediments. Compactification of the higher-dimensional theories is used to produce four-dimensional SUSY theories with $\mathcal{N}=2$ and $\mathcal{N}=4$ extended supersymmetries. The $\mathcal{N}=4$ theory is particularly interesting as it provides the first perturbed version of the conformal field theory that may have a simple Ads/CFT dual.

\footnotetext{
* Also at Division of Natural Sciences, New College of Florida.

$\dagger$ mcdonald@ncf.edu
}

\section{CPT PRESERVING MODEL}

The CPT-preserving model that violates Lorentz symmetry is particularly simple to implement as there exists a modified superspace implementation in terms of $Q$ operators. This is not the case for the CPT-violating model. Introduce the twisted derivative operator [3]

$$
\tilde{\partial}_{\mu}=\partial_{\mu}+k_{\mu}^{\nu} \partial_{\nu}
$$

the general vector superfield is expanded in terms of the twisted derivative as

$$
\begin{aligned}
V(x, \theta)= & C(x)+i \bar{\theta} \gamma^{5} \omega(x)-\frac{i}{2} \bar{\theta} \gamma^{5} \theta M(x)-\frac{1}{2} \bar{\theta} \theta N(x) \\
& +\frac{1}{2} \bar{\theta} \gamma^{5} \gamma^{\mu} \theta \tilde{V}_{\mu}-i \bar{\theta} \gamma^{5} \theta \bar{\theta}\left[\lambda(x)+\frac{i}{2} \tilde{\partial} \omega(x)\right] \\
& +\frac{1}{4}(\bar{\theta} \theta)^{2}\left(D(x)-\frac{1}{2} \tilde{\partial}_{\mu} \tilde{\partial}^{\mu} C(x)\right),
\end{aligned}
$$

where $\tilde{V}_{\mu}=V_{\mu}+k_{\mu \nu} V^{\nu}$ is inserted into the expansion. This turns out to be necessary to preserve conventional gauge invariance in the resulting supersymmetric lagrangian. It is also consistent with imposing a shift of the entire covariant derivative operator rather than simply the partial derivative part. The supersymmetry transformations are implemented using the operator $\delta_{Q} V(x, \theta)=-i \bar{\epsilon} Q V(x, \theta)$, where

$$
Q=i \partial_{\bar{\theta}}-\gamma^{\mu} \theta \tilde{\partial}_{\mu} .
$$

Note that this operator satisfies the perturbed anticommuation relation

$$
\{Q, \bar{Q}\}=2 \gamma^{\mu}\left(P_{\mu}+k_{\mu \nu} P^{\nu}\right) .
$$

Matching terms in the conventional way yields the SUSY transformation properties of the fields. The ones that will be useful in the present work are

$$
\begin{aligned}
\delta \lambda & =\frac{i}{2} \sigma^{\mu \nu} \tilde{F}_{\mu \nu} \epsilon-i \gamma^{5} D \epsilon, \\
\delta \tilde{V}^{\mu} & =-i \bar{\epsilon}\left(\gamma^{\mu} \lambda+i \tilde{\partial}^{\mu} \omega\right), \\
\delta D & =\bar{\epsilon} \tilde{\partial} \gamma^{5} \lambda,
\end{aligned}
$$


where the quantity $\tilde{F}$ is a twisted field strength (note that $\tilde{F}$ does not have the conventional meaning of dual in this manuscript) given by

$$
\tilde{F}^{\mu \nu}=\tilde{\partial}^{\mu} \tilde{V}^{\nu}-\tilde{\partial}^{\nu} \tilde{V}^{\mu}
$$

Note that the $D$-term still transforms as a total derivative and can therefore be used to construct SUSY lagrangians.

A general super gauge transformation is implemented by adding a chiral superfield expressed in terms of the twisted derivative. The result is the vector superfield in the Wess-Zumino gauge

$$
V(x, \theta)=\frac{1}{2} \bar{\theta} \gamma^{5} \gamma^{\mu} \theta \tilde{V}_{\mu}-i \bar{\theta} \gamma^{5} \theta \bar{\theta} \lambda(x)+\frac{1}{4}(\bar{\theta} \theta)^{2} D(x)
$$

Note that a twisted gauge transformation on $\tilde{V}_{\mu}$ results in symmetry under the conventional gauge transformation for $V^{\mu}$

$$
V^{\mu} \rightarrow V^{\mu}+\partial^{\mu} \Lambda
$$

This is the reason that the original superfield was expanded in terms of $\tilde{V}^{\mu}$ rather than $V^{\mu}$. The supercovariant derivative can be used to calculate the spinor superfield $W_{\alpha}=1 / 4 \overline{\mathcal{D}} \gamma^{5} \mathcal{D} \mathcal{D}_{\alpha} V$, with $\mathcal{D}=i \partial_{\bar{\theta}}+\gamma^{\mu} \theta \tilde{\partial}_{\mu}$. The left component of $W$ is

$$
\begin{aligned}
W_{L}= & \lambda_{L}\left(x_{+}\right)-\frac{1}{2} \gamma^{\mu} \gamma^{\nu} \theta_{L} \tilde{F}_{\mu \nu}\left(x_{+}\right)-i \bar{\theta}_{R} \theta_{L} \tilde{\not} \lambda_{R}\left(x_{+}\right) \\
& -i \theta_{L} D\left(x_{+}\right)
\end{aligned}
$$

where the left-chiral coordinate $x_{+}$is given by

$$
x_{+}^{\mu}=x^{\mu}+\frac{i}{2} \bar{\theta} \gamma^{5} \gamma^{\mu} \theta+\frac{i}{2} k^{\mu \nu} \bar{\theta} \gamma^{5} \gamma_{\nu} \theta
$$

The lagrangian invariant under the modified SUSY transformation is constructed using the $\theta^{2}$-term in the secondorder function

$$
\mathcal{L}=\frac{1}{2} \operatorname{Re}\left(\left.\bar{W}_{L} \gamma^{5} W_{L}\right|_{\theta^{2}}\right)=-\frac{1}{4} \tilde{F}^{2}+\frac{i}{2} \bar{\lambda} \tilde{\partial} \lambda+\frac{1}{2} D^{2} .
$$

Note that this construction works because the leftsuperfield satisfies the twisted chirality condition

$$
\mathcal{D}_{R} W_{L}=0
$$

where $\mathcal{D}_{R}=1 / 2\left(1+\gamma^{5}\right) \mathcal{D}$ is the right-handed projection of the twisted supercovariant derivative operator. Any function of $W_{L}$ therefore satisfies the twisted chirality condition and hence leads to a SUSY covariant $\theta^{2}$-term, or ' $F$-Term' as it is commonly denoted. Explicit verification of this SUSY requires the use of the twisted Jacobi Identity

$$
\epsilon^{\mu \nu \alpha \beta} \tilde{\partial}_{\nu} \tilde{F}_{\alpha \beta}=0
$$

satisfied by the twisted field strength due to simple symmetry principles. This Lagrangian can be put into the form of the Standard Model Extension (SME) Parameters by expanding the Lagrangian in the form

$$
\begin{aligned}
\mathcal{L}= & -\frac{1}{4}\left(F^{2}+k_{F}^{\mu \nu \alpha \beta} F_{\mu \nu} F_{\alpha \beta}\right)+\frac{i}{2}\left(\bar{\lambda} \gamma^{\mu} \partial_{\mu} \lambda+\bar{\lambda} c_{\mu \nu} \gamma^{\mu} \partial^{\nu} \lambda\right) \\
& +\frac{1}{2} D^{2},
\end{aligned}
$$

where

$$
\begin{aligned}
k_{F}^{\mu \nu \alpha \beta}= & 2\left(2 k^{\alpha \mu}+\left(k^{2}\right)^{\alpha \mu}\right) g^{\beta \nu}+4\left(k^{\mu \alpha}+\left(k^{2}\right)^{\alpha \mu}\right) k^{\nu \beta} \\
& +\left(k^{2}\right)^{\alpha \mu}\left(k^{2}\right)^{\beta \nu}
\end{aligned}
$$

and

$$
c_{\mu \nu}=k_{\mu \nu}
$$

In this expression, $k^{2}$ represents the square of the matrix for $k$

$$
\left(k^{2}\right)^{\mu \nu}=k^{\mu \alpha} k_{\alpha}^{\nu} .
$$

Note that in contrast to the scalar chiral superfield model where only second-order terms in $k$ were present, thirdand fourth-order terms in $k$ appear in the vector superfield Lagrangian.

\section{ATTEMPT AT CONSTRUCTION OF A CPT VIOLATING MODEL}

CPT violation can be incorporated into the chiral superfield using a phase redefinition of the fields involved [3]. When this same procedure is adopted for the vector superfield case, several problems emerge. Suppose that the fermion field is redefined by

$$
\tilde{\lambda}=e^{-i \gamma^{5} k \cdot x} \lambda
$$

to induce CPT-violating terms as is done in the chiral superfield case. The phase cannot be absorbed into the real vector field $V_{\mu}$ as can be seen from the following decomposition of the vector field supersymmetry transformation

$$
\delta V^{\mu}=-i \bar{\epsilon} \gamma^{\mu}\left(e^{-i k \cdot x} \lambda_{R}+e^{i k \cdot x} \lambda_{L}\right) .
$$

This expression implies that it is impossible to absorb both of the phase changes into a single redefinition of the vector field as was possible in the chiral superfield case. This indicates that the CPT-violating generalization of the vector superfield probably does not exist.

\section{IV. $\mathcal{N}=2$ FROM SIX-DIMENSIONAL THEORY}

The extension to higher dimensions and the following compactification process uses the notation and techniques introduced in [4]. The definitions of $L$ and $R$ are switched relative to the first half of this paper, this notation is kept in order to provide an easier comparison with the unperturbed results of [4]. 
The supersymmetric Lagrangian (13) may be extended to six dimensions by allowing the $\gamma$ matrices and vector field indices to run from 0 to 6 and setting the auxiliary field $D$ to zero. It is not possible to impose the Majorana condition in six dimensions, but it is possible to impose the Weyl condition. The Lagrangian

$$
\mathcal{L}=-\frac{1}{4} \tilde{F}^{2}+i \bar{\lambda} \tilde{\partial} \lambda .
$$

is supersymmetric under the modified supersymmetry transformations

$$
\begin{aligned}
\delta \lambda & =\frac{i}{2} \sigma^{\mu \nu} \tilde{F}_{\mu \nu} \epsilon, \\
\delta \tilde{V}^{\mu} & =-i\left(\bar{\epsilon} \Gamma^{\mu} \lambda-\bar{\lambda} \Gamma_{\mu} \epsilon\right) .
\end{aligned}
$$

The gamma matrices are chosen in a basis where $\Gamma^{\mu}=$ $\gamma^{\mu} \otimes I_{2}$ for $\mu=0,1,2,3$ and

$$
\begin{gathered}
\Gamma^{4,5}=\gamma^{5} \otimes i \sigma^{1,2}, \\
\Gamma^{7}=\gamma^{5} \otimes \sigma^{3} .
\end{gathered}
$$

In this basis, the Weyl condition fixes $\lambda$ to have the structure

$$
\lambda=\left(\begin{array}{c}
L \chi \\
R \chi
\end{array}\right)
$$

where $\chi$ is a four-dimensional Dirac spinor. The components of the vector potential remain the same for $\mu=0,1,2,3$, while $A_{4}=S, A_{5}=P$, are relabeled as scalar and pseudoscalar fields. It is convenient to investigate special cases for $k^{\mu \nu}$. The first case is when the lorentz violation is restricted to lie in the four physical dimensions $\left(k^{\mu 4}=k^{\mu 5}=0\right)$. The conventional dimensional reduction procedure yields the Lagrangian

$$
\mathcal{L}=-\frac{1}{4} \tilde{F}^{2}+\frac{1}{2} \tilde{\partial}_{\mu} S \tilde{\partial}^{\mu} S+\frac{1}{2} \tilde{\partial}_{\mu} P \tilde{\partial}^{\mu} P+i \bar{\chi} \tilde{\partial} \chi .
$$

This lagrangian has an $\mathcal{N}=2$ supersymmetry under the transformation laws

$$
\begin{aligned}
\delta \tilde{V}^{\mu} & =i\left(\bar{\alpha} \gamma_{\mu} \chi-\bar{\chi} \gamma_{\mu} \alpha\right), \\
\delta P & =\bar{\chi} \gamma^{5} \alpha-\bar{\alpha} \gamma^{5} \chi, \\
\delta S & =i(\bar{\chi} \alpha-\bar{\alpha} \chi), \\
\delta \chi & =\left(\frac{i}{2} \sigma^{\mu \nu} \tilde{F}_{\mu \nu} \alpha+i \not \tilde{D} P \gamma^{5}-\tilde{\partial} S\right) \alpha .
\end{aligned}
$$

Another interesting special case is when the Lorentz violation is restricted to the compactified dimensions. In this case, the gauge and fermion terms are unperturbed while the $S$ and $P$ fields are mixed by the $k^{\mu \nu}$ parameters according to

$$
\begin{aligned}
\tilde{S} & =\left(1-k_{44}\right) S-k_{45} P, \\
\tilde{P} & =\left(1-k_{55}\right) P-k_{45} S .
\end{aligned}
$$

The lagrangian in this case is the usual one with the replacements $S \rightarrow \tilde{S}$ and $P \rightarrow \tilde{P}$. In the free theory this is just a redefinition of fields, but if the theory is coupled to other sectors that involve the $S U(2)$ symmetry, there could be observable effects of the violation.

\section{V. $\mathcal{N}=4$ FROM TEN-DIMENSIONAL THEORY}

The supersymmetric Lagrangian (13) may be extended to ten dimensions by allowing the $\gamma$ matrices and vector field to have indices running from 0 to 9 and setting the auxiliary field $D$ to zero

$$
\mathcal{L}=-\frac{1}{4} \tilde{F}^{2}+\frac{i}{2} \bar{\lambda} \tilde{\partial} \lambda
$$

The gamma matrices are $32 \times 32$ matrices in this dimension and are denoted using $\Gamma^{\mu}$. The first four ( $\mu=0,1,2,3)$ are chosen to reduce to the usual fourdimensional gamma matrices following compactification $\Gamma^{\mu}=\gamma^{\mu} \otimes I_{8}$. The rest of the gamma matrices are defined as linear combinations of a more convenient basis selected for its $\mathrm{SU}(4)$ properties

$$
\begin{array}{ll}
\Gamma^{4}=\Gamma^{14}+\Gamma^{23}, & i \Gamma^{7}=\Gamma^{14}-\Gamma^{23}, \\
\Gamma^{5}=\Gamma^{24}-\Gamma^{13}, & i \Gamma^{8}=\Gamma^{24}+\Gamma^{13}, \\
\Gamma^{6}=\Gamma^{34}+\Gamma^{12}, & i \Gamma^{9}=\Gamma^{34}-\Gamma^{12} .
\end{array}
$$

where

$$
\Gamma^{i j}=\gamma_{5} \otimes\left(\begin{array}{cc}
0 & \rho^{i j} \\
\rho_{i j} & 0
\end{array}\right),
$$

are written in terms of the $4 \times 4$ matrices

$$
\left(\rho^{i j}\right)_{k l}=\delta_{i k} \delta_{j l}-\delta_{j k} \delta_{i l},
$$

and

$$
\left(\rho_{i j}\right)_{k l}=\frac{1}{2} \epsilon_{i j m n}\left(\rho^{m n}\right)_{k l}=\epsilon_{i j k l} .
$$

The product of all of the gamma matrices defines

$$
\Gamma^{11}=\gamma^{5} \otimes\left(\begin{array}{cc}
I_{4} & 0 \\
0 & -I_{4}
\end{array}\right),
$$

and the charge conjugation matrix is

$$
C^{10}=C \otimes\left(\begin{array}{cc}
0 & I_{4} \\
I_{4} & 0
\end{array}\right)
$$

where $C$ is the usual four-dimensional charge conjugation matrix. The spinor is required to be an eigenstate of both $\Gamma^{11}$ and $C_{10}$ therefore imposing the Majorana and Weyl conditions as in the standard case.

The previous construction of the perturbed theory still works, provided that the twisted Jacobi Identity is modified appropriately to

$$
A^{\nu \alpha \beta} \tilde{\partial}_{\nu} \tilde{F}_{\alpha \beta}=0,
$$

where $A^{\nu \alpha \beta}$ is a totally antisymmetric 3 -tensor in $10 \mathrm{di}-$ mensions. It is then possible to compactify the extra six dimensions onto a torus [4] and obtain a perturbed $N=4$ supersymmetric model that violates Lorentz Invariance through the twisted derivative. Any Lorentz violation 
present in the extra dimensions will show up as a violation of the $S U(4)$ symmetry. Such a model should be interesting from the perspective of Ads/CFT theory as it provides the first example of an appropriate Lorentzviolating theory that can be used in this context. In fact, standard dimensional reduction procedures may be used to produce different SUSY theories in four dimensions that may or may not violate Lorentz invariance in the four physical dimensions.

For example, The $N=4$ SUSY model with Lorentz violation takes the form

$$
\mathcal{L}=-\frac{1}{4} \tilde{F}^{2}+i \bar{\chi}_{i} \tilde{\partial} L \chi^{i}+\frac{1}{4} \tilde{\partial}_{\mu} \phi_{i j} \tilde{\partial}^{\mu} \phi^{i j},
$$

where the $k_{\mu \nu}$ background tensor was chosen to vanish along the compactified dimensions, but be of general form in the remaining four dimensions. The complex scalar fields $\phi_{i j}$ transform as a 6 of $S U(4)$ and are defined according to

$$
\phi_{i 4}=\frac{1}{\sqrt{2}}\left(A_{i+3}+i A_{i+6}\right), \quad i=1,2,3,
$$

and

$$
\phi^{j k}=\frac{1}{2} \epsilon^{j k l m} \phi_{l m}=\left(\phi_{j k}\right)^{*},
$$

and the fermions $\chi^{i}$ form a 4 representation of $S U(4)$. The modified SUSY transformation laws of the fields are

$$
\begin{aligned}
\delta \tilde{A}_{\mu} & =-i\left(\bar{\alpha}_{i} \gamma_{\mu} L \chi^{i}-\bar{\chi}_{i} \gamma_{\mu} L \alpha^{i}\right) \\
\delta \phi_{i j} & =-\sqrt{2} i\left(\bar{\alpha}_{j} R \tilde{\chi}_{i}-\bar{\alpha}_{i} R \tilde{\chi}_{j}+\epsilon_{i j k l} \overline{\tilde{\alpha}}^{k} L \chi^{l}\right) \\
\delta L \chi^{i} & =\frac{i}{2} \sigma^{\mu \nu} \tilde{F}_{\mu \nu} L \alpha^{i}-\sqrt{2} \gamma^{\mu} \tilde{\partial}_{\mu} \phi^{i j} R \tilde{\alpha}_{j} \\
\delta R \tilde{\chi}_{i} & =\frac{i}{2} \sigma^{\mu \nu} \tilde{F}_{\mu \nu} R \tilde{\alpha}_{i}+\sqrt{2} \gamma^{\mu} \tilde{\partial}_{\mu} \phi_{i j} L \alpha^{j}
\end{aligned}
$$

Perturbations appear in all three sectors and they are connected by the requirement of supersymmetry.

Another interesting special case occurs when the Lorentz-violating coefficients are taken to vanish along the four physical dimensions, but are allowed to be arbitrary in the compactified dimensions. In this case, the four-dimensional theory is Lorentz invariant, but violates $S U(4)$ symmetry. The perturbations all appear in the scalar sector as the resulting Lagrangian takes the form

$$
\mathcal{L}=-\frac{1}{4} F^{2}+i \bar{\chi}_{i} \not \partial L \chi^{i}+\frac{1}{4} \partial_{\mu} \tilde{\phi}_{i j} \partial^{\mu} \tilde{\phi}^{i j},
$$

where $\tilde{\phi}_{i j}=\phi_{i j}+\Lambda_{i j k l} \phi_{k l}$ and the matrix $\Lambda^{i j k l}$ contains the induced Lorentz violation from the higher dimensions. The supersymmetry transformations for these fields are

$$
\begin{aligned}
\delta A_{\mu} & =-i\left(\bar{\alpha}_{i} \gamma_{\mu} L \chi^{i}-\bar{\chi}_{i} \gamma_{\mu} L \alpha^{i}\right) \\
\delta \tilde{\phi}_{i j} & =-\sqrt{2} i\left(\bar{\alpha}_{j} R \tilde{\chi}_{i}-\bar{\alpha}_{i} R \tilde{\chi}_{j}+\epsilon_{i j k l} \overline{\tilde{\alpha}}^{k} L \chi^{l}\right) \\
\delta L \chi^{i} & =\frac{i}{2} \sigma^{\mu \nu} F_{\mu \nu} L \alpha^{i}-\sqrt{2} \gamma^{\mu} \partial_{\mu} \tilde{\phi}^{i j} R \tilde{\alpha}_{j} \\
\delta R \tilde{\chi}_{i} & =\frac{i}{2} \sigma^{\mu \nu} F_{\mu \nu} R \tilde{\alpha}_{i}+\sqrt{2} \gamma^{\mu} \partial_{\mu} \tilde{\phi}_{i j} L \alpha^{j} .
\end{aligned}
$$

In this case, identification of $\tilde{\phi}$ with the physical scalar fields removes any effect of the higher-dimensional Lorentz violation and restores $S U(4)$ symmetry. However, this is only possible if the fields $\phi_{i j}$ do not couple with other sectors as their redefinition would affect their symmetry properties in relation to the other sectors. If this is the case, the above model demonstrates a possible method by which the $S U(4)$ symmetry of an N=4 supersymmetry model may be broken. Some fields can be driven to irrelevant status by making their kinetic coupling very small. Note that the matrix $\Lambda^{i j k l}$ in this model does not in fact have to be small since it does not induce Lorentz violation in the four physical dimensions.

\section{NON ABELIAN GENERALIZATION}

The above constructions also work if the full covariant derivative is twisted by $k^{\mu \nu}$ according to

$$
\tilde{D}^{\mu}=D^{\mu}+k^{\mu \nu} D_{\nu} .
$$

For example, the non-Abelian ten-dimensional theory then takes the form

$$
\mathcal{L}=\operatorname{Tr}\left[-\frac{1}{2} \tilde{F}^{2}+i \bar{\lambda} \tilde{D} \lambda\right],
$$

where $i g \tilde{F}^{\mu \nu}=\left[\tilde{D}^{\mu}, \tilde{D}^{\nu}\right]$, and the supersymmetry transformations are the same as before written in terms of $\tilde{F}$. The key fact that allows the supersymmetry transformation to succeed is the Bianchi Identity satisfied by $\tilde{F}^{\mu \nu}$

$$
\left[\tilde{D}^{\alpha},\left[\tilde{D}^{\beta}, \tilde{D}^{\gamma}\right]\right]+\left[\tilde{D}^{\beta},\left[\tilde{D}^{\gamma}, \tilde{D}^{\alpha}\right]\right]+\left[\tilde{D}^{\gamma},\left[\tilde{D}^{\alpha}, \tilde{D}^{\beta}\right]\right]=0 .
$$

The first case considered is when the Lorentz-violating couplings $k^{\mu \nu}$ are restricted to the four physical dimensions. The resulting Lagrangian is

$$
\begin{aligned}
\mathcal{L}= & \operatorname{Tr}\left[-\frac{1}{2} \tilde{F}^{2}+2 i \bar{\chi}_{i} \tilde{D} L \chi^{i}\right. \\
& +\sqrt{2} g\left(\overline{\tilde{\chi}}\left[\phi_{i j}, L \chi^{j}\right]-\bar{\chi}\left[\phi^{i j}, R \tilde{\chi}_{j}\right]\right) \\
& \left.+\frac{1}{2} \tilde{D}_{\mu} \phi_{i j} \tilde{D}^{\mu} \phi^{i j}+\frac{g^{2}}{8}\left[\phi_{i j}, \phi_{k l}\right]^{2}\right] .
\end{aligned}
$$

The case when $k^{\mu \nu}$ is restricted to the compactified dimensions is simplest, the theory is the same as the conventional one with the replacement

$$
\tilde{\phi}_{i j}=\phi_{i j}+\Lambda_{i j k l} \phi_{k l},
$$

with lagrangian

$$
\begin{aligned}
\mathcal{L}= & \operatorname{Tr}\left[-\frac{1}{2} F^{2}+2 i \bar{\chi}_{i} D L \chi^{i}\right. \\
& +\sqrt{2} g\left(\overline{\tilde{\chi}}\left[\tilde{\phi}_{i j}, L \chi^{j}\right]-\bar{\chi}\left[\tilde{\phi}^{i j}, R \tilde{\chi}_{j}\right]\right) \\
& \left.+\frac{1}{2} D_{\mu} \tilde{\phi}_{i j} D^{\mu} \tilde{\phi}^{i j}+\frac{g^{2}}{8}\left[\tilde{\phi}_{i j}, \tilde{\phi}_{k l}\right]^{2}\right] .
\end{aligned}
$$




\section{NOTE ON COORDINATE TRANSFORMATIONS}

An interesting question arises as to the nature of the $k^{\mu \nu}$ model regarding physical observability due to possible coordinate transformations that can alter, or remove Lorentz-Violating terms [5]. To answer this question, one starts with the conventional Lagrangian with no symmetry violating terms in it and performs the appropriate change in coordinates for

$$
\partial^{\prime \mu}=\partial^{\mu}+k^{\mu}{ }_{\nu} \partial^{\nu}
$$

corresponding to the derivative replacement used in the SUSY-LV formulas. The initial lagrangian is written in the form

$$
\mathcal{L}_{0}=-\frac{1}{4} g^{\mu \alpha} g^{\nu \beta} F_{\mu \nu} F_{\alpha \beta}+\frac{i}{2} g^{\mu \nu} \bar{\lambda} \gamma_{\mu} \partial_{\nu} \lambda+\frac{1}{2} D^{2}
$$

where the metric $g^{\mu \nu}=\eta^{\mu \nu}$ is exhibited explicitly. This form of the SUSY lagrangian is written in geometric form so that it is covariant under the linear coordinate transformation consistent with Eq. (63),$x^{\mu}=x^{\mu}-k^{\mu}{ }_{\nu} x^{\prime \nu}$. A simple calculation to lowest-order in $k^{\mu \nu}$ yields a modified metric

$$
g^{\prime \mu \nu}=\eta^{\mu \nu}+2 k^{\mu \nu},
$$

and the corresponding lagrangian in the new coordiates reads the same as Eq. (64) with primes on all of the quantities involved, including the gamma matrices which satisfy the modified Clifford algebra relation

$$
\left\{\gamma^{\prime \mu}, \gamma^{\prime \nu}\right\}=2 g^{\prime \mu \nu}=2 \eta^{\mu \nu}+4 k^{\mu \nu} .
$$

Expanding the $g^{\prime}$ factors and expressing the primed gamma in terms of the conventional gamma yields the Lagrangian

$$
\begin{aligned}
\mathcal{L}= & -\frac{1}{4}\left(\eta^{\mu \alpha} \eta^{\nu \beta} F_{\mu \nu}^{\prime} F_{\alpha \beta}^{\prime}+k_{F}^{\mu \nu \alpha \beta} F_{\mu \nu}^{\prime} F_{\alpha \beta}^{\prime}\right) \\
& +\frac{i}{2}\left(\bar{\lambda}^{\prime} \eta^{\mu \nu} \gamma_{\mu} \partial_{\nu}^{\prime} \lambda^{\prime}+\bar{\lambda}^{\prime} k_{\mu \nu} \gamma^{\mu} \partial^{\prime \nu} \lambda^{\prime}\right)+\frac{1}{2} D^{\prime 2} .
\end{aligned}
$$

This lagrangian is formally equivalent to the supersymmetric Lorentz-breaking theory Eq. ( [16), however, the vectors and spinors are resolved in a non-orthogonal coordinate system This means that all manipulations using the transformed lagrangian must take into account this different property of the vector indices. For example, quantities like

$$
A^{\prime^{\mu}} A_{\mu}^{\prime}=g^{\prime \mu \nu} A_{\mu}^{\prime} A_{\nu}^{\prime}=\eta^{\mu \nu} A_{\mu}^{\prime} A_{\nu}^{\prime}+2 k^{\mu \nu} A_{\mu}^{\prime} A_{\nu}^{\prime} .
$$

must be used to compute lengths of vectors in the transformed system. Since the assumed metric in Eq. ( 16) is simply $\eta^{\mu \nu}$, no such complication in dealing with vector components arises, so the theory is physically distinct from a conventional theory expressed in skewed coordinates, provided that the metric is accessible to the experimentalist through using rods and clocks constructed of other, conventional Lorentz invariant fields.

Another way to put this is that it is possible to change coordinates to remove $k^{\mu \nu}$ in the Lagrangian, but it will re-appear in the vector coordinate manipulations as a pesky non-orthogonality of the vectors (and spinors) involved in the calculations of physical observables. It will also appear in any Lorentz-invariant sectors as a new perturbation in those fields. This argument also works provided there are at least two sectors with different valued $k_{\mu \nu}$ tensors present in the theory.

\section{SUMMARY}

The main result of this paper is that the scalar CPTpreserving models with Lorentz Violation easily generalize to the vector superfield and can be extended to the usual higher-dimensional cases. In addition, compactification can be used to construct extended supersymmetry models in four-dimensions. The theory may be abelian, or non-abelian, the same basic technique works for both cases. On the other hand, the scalar CPT-violating models do not easily generalize to the vector superfield. The reason for this is connected with the inability to separate the vector potential into left- and right-handed components.

The new $\mathcal{N}=4$ theories with Lorentz violation are particularly interesting from a theoretical viewpoint as the conventional theory plays a crucial role in Ads/CFT theories. One of the main purposes of this paper is to construct the perturbed $\mathcal{N}=4$ theories so that they may be studied in this context.
[1] S. Nibbelink and M. Pospelov, Phys. Rev. Lett. 94, 081601 (2005).

[2] H. Belich, G. Dias, J. Helayel-Neto, F. Leal, and W. Spalenza(2010), arXiv:1009.1326.

[3] M. Berger and A. Kostelecký, Phys. Rev. D 65, 091701 (2002).
[4] L. Brink, J. Schwartz, and J. Scherk, Nucl. Phys. B 121, 77 (1977).

[5] D. Colladay and P. McDonald, J. Math. Phys. 43, 3554 (2002). 